\title{
Algunos determinantes de la movilidad estudiantil universitaria de los países andinos
}

\author{
Alan Fairlie Reinoso*
}

\section{Resumen}

El artículo destaca la movilidad estudiantil como un eje fundamental de la internacionalización de la educación superior, y los factores que propician el flujo de estudiantes de educación superior desde países andinos hacia países desarrollados. Para ello, se estima un modelo gravitacional con el objetivo de encontrar dichos determinantes, entre los que destacan: el ingreso en el país de destino, el idioma, y las barreras regulatorias (tomando como proxi un índice de libertad económica). Esto sugiere, que convenios con países donde hay más apertura y menores regulaciones, favorecerían la movilidad. Pero, es necesario identificar otros obstáculos que deberían ser removidos, en los convenios que hagan las instituciones o las negociaciones que lideren los países.

Palabras clave: Internacionalización, movilidad estudiantil, educación superior, países desarrollados, países andinos

\begin{abstract}
The article highlights student mobility as an essential's axis of the internationalization of higher education, also identify the factors that are in favor the flow of higher education students from developing countries to developed countries. Besides, had established main factors that influence the selection of the country destination from Andean's country students to developed countries are determined.

We estimated a gravity model, to find some determinants of this, and the most important are the income in the destination country, the language, and regulatory barriers (taking as a proxy, the index of economic freedom). This suggests that agreements with countries where there is more openness and less regulations, would favors mobility. But it is necessary to identify other obstacles that should be removed, in the agreements between countries or institutions.
\end{abstract}

Keywords: Internationalization, student mobility, higher education, Developed countries, Andean countries

\footnotetext{
* Director de la Maestría en Biocomercio y Desarrollo Sostenible, Pontificia Universidad Católica del Perú (PUCP). Parlamentario Andino, Presidente de la Comisión Cuarta del Parlamento Andino. Decano de la Facultad de Ciencias Sociales, PUCP. Doctor en Gestión Estratégica con mención en Innovación y Gestión en Educación Superior, Pontificia Universidad Católica del Perú. Email: afairli@pucp.edu.pe. Recibido: 10 de diciembre de 2019; modificaciones: 18 de marzo de 2021; aceptado. 09 de abril de 2021.
} 
Alan Fairlie Reinoso

Algunos determinantes de la movilidad estudiantil universitaria de los países andinos

\section{Introduction}

Con la globalización, los procesos de internacionalización de educación superior tendieron a masificarse, y en ese contexto la movilidad académica se ha convertido en una de las principales estrategias que propicia la integración de la dimensión internacional, en los aprendizajes. Además, se plantea como un medio para mejorar el prestigio y visibilidad de las universidades, convirtiéndose en una estrategia institucional (IESALC, 2019).

Por otro lado, la movilidad académica tiene un papel fundamental dentro de las agendas nacionales en materia de educación superior. Así, diversos gobiernos promueven actividades que facilitan la movilidad de estudiantes, profesores e investigadores, ya sea a través de programas de cooperación bilateral, o multilaterales.

En ese sentido, el éxito o fracaso de los procesos de internacionalización no solo dependerán de las políticas impulsadas desde las universidades, sino de la normativa del sistema universitario, la estrategia educativa del país, y los compromisos de liberalización de servicios educativos en los foros internacionales.

Una forma de visualizar la clasificación de los procesos de internacionalización es con el marco que los países definieron en la OMC (OMC, 2015), en donde se estableció una tipología de internacionalización de los servicios en cuatro modos de prestación, que aplicadas a la educación se refieren a: la movilidad docente y estudiantil, la movilidad de instituciones, la educación transfronteriza, y las redes de investigación.

Este artículo, busca determinar los principales factores que influyen en la movilidad de los estudiantes de educación superior de los países de la región Andina, uno de los instrumentos principales en sus procesos de internacionalización. Se parte de la definición de internacionalización, para luego analizar las modalidades existentes, centrándonos en la movilidad de estudiantes y las variables asociadas a esta. Asimismo, se hace un breve balance de la situación actual de la movilidad estudiantil en América Latina. Se presentan las principales barreras al comercio de servicios de educación superior, y el reto que implica en los procesos de internacionalización. Finalmente, realizamos una estimación empírica, con un modelo de gravedad que identifica algunos de los factores que promueven la movilidad de estudiantes andinos hacia países desarrollados.

\section{Modalidades de la internacionalización de la educacion superior}

Existen diversas definiciones sobre la internacionalización y diferentes enfoques relacionados a este proceso. Para autores como Rama (2014), la internacionalización educativa es un proceso global en expansión al interior de un sistema económico mundial, e inmerso en una diversificación de sus dimensiones y áreas bajo las cuales se expresa. Estas son las partes constitutivas, las articulaciones del sistema educativo global, las cuales muestran las características diferenciadas de los sistemas nacionales. Por su parte, Knight (2003) sostiene que la internacionalización a nivel nacional, sectorial e institucional se define como: "el proceso de integración de una dimensión internacional, intercultural o global en el propósito y funciones de la provisión de educación postsecundaria” (p.2).

Asimismo, Gacel-Ávila y Rodríguez-Rodríguez (2018a), sostienen que la internacionalización es "una de las tendencias clave para transformar la educación terciaria en todo el mundo y adaptarla a las 
demandas de una sociedad global, multicultural y altamente competitiva” (p.57), por lo que resulta estratégico que las instituciones educativas implementen estrategias para sus procesos de internacionalización.

La educación superior se ha inclinado cada vez más por lo internacional, surgiendo nuevas dimensiones como las descritas por Rama (2014): la movilidad estudiantil, la movilidad docente, apertura de sedes universitarias en países extranjeros, impulso de la educación virtual convirtiéndose en una educación transfronteriza; fomento del aseguramiento de la calidad de los aprendizajes por medio de los licenciamientos institucionales y las acreditaciones de los programas, internacionalización del currículo y la movilidad o la migración profesional.

\section{Gráfico 1: Las distintas internacionalizaciones}

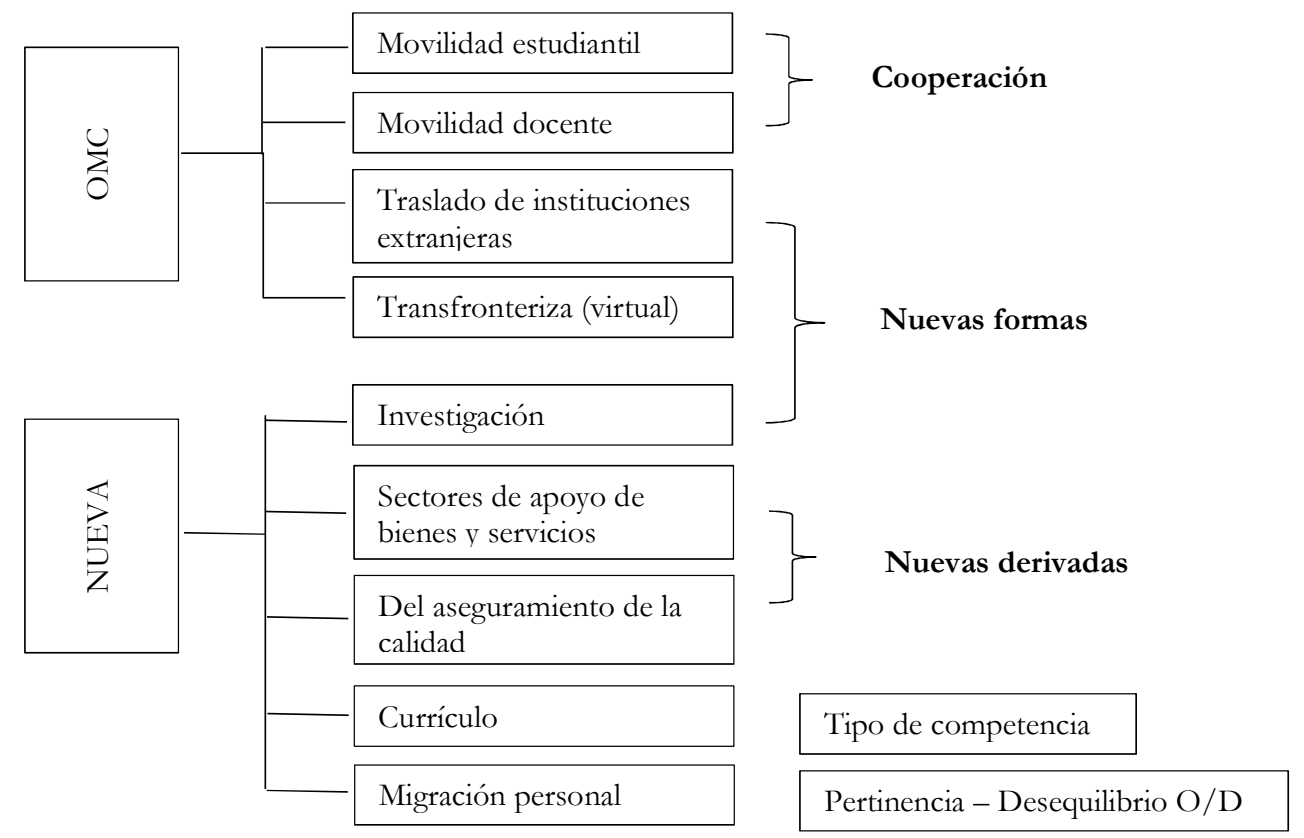

Fuente: Rama (2014)

Las modalidades de internacionalización de la educación superior variarán de acuerdo con las causas internas que las originan, así como a las asimetrías que definen la relación entre los países más y menos desarrollados. Estas asimetrías, pueden ser en términos de calidad, diversidad, pertinencia, equidad y cobertura de los sistemas nacionales, por lo cual resultan ser un factor esencial para promover los procesos de internacionalización, puesto que impulsan la demanda por una formación de calidad que usualmente no se logra dar dentro del sistema local y/o nacional.

En ese sentido, los diversos procesos de internacionalización de la educación superior se han ido consolidando a través de estrategias y acciones desarrolladas principalmente por las universidades, entre las que destaca la movilidad estudiantil, ya que esta es considerada por muchos autores como una de las tendencias más importantes; puesto que, no solo mejora la formación integral de los estudiantes, sino que incorpora una visión cultural, académica y técnica internacional dentro de sus procesos de aprendizajes (Theiler, 2019). 
Alan Fairlie Reinoso

Algunos determinantes de la movilidad estudiantil universitaria de los países andinos

\section{La movilidad estudiantil como parte de la internacionalización de la educación superior}

\section{Movilidad estudiantil}

La globalización, así como el avance de las sociedades de conocimiento, originaron el surgimiento de iniciativas orientadas a la conformación de espacios comunes de educación superior, basados en la armonización y la convergencia de los sistemas de educación superior, como es el caso del "Proceso de Bolonia", en donde se establecen parámetros para adaptar y armonizar progresivamente los sistemas de educación superior. Es así que, procura dar mayor coherencia a los sistemas de educación superior en Europa, además de impulsar la movilidad de estudiantes y personal, para lo cual acordaron:

Introducir un sistema de educación superior de tres ciclos (grado, máster y doctorado); garantizar el reconocimiento mutuo de las cualificaciones y los períodos de aprendizaje en el extranjero completados en otras universidades; y aplicar un sistema de garantía de la calidad, a fin de reforzar la calidad y pertinencia del aprendizaje y la enseñanza (UE, 2021).

De esta forma, se busca propiciar una educación superior inclusiva y accesible, además de ser más atractiva y competitiva a nivel mundial.

Asimismo, el Proceso de Bolonia impulsó la movilidad estudiantil y de investigadores buscando superar los factores que dificultan dicha movilidad, en consonancia con el programa Erasmus. Este programa ha estado orientado a promover la educación superior europea, además de apoyar la cooperación con terceros países. Erasmus ofrece apoyo financiero a las instituciones y becas para los estudiantes, así como movilidad académica de personal administrativo y docente, convirtiéndose de este modo en un referente de programa de movilidad estudiantil a nivel global.

Es así como el Proceso de Bolonia, ha influenciado para que otras iniciativas de armonización regional en materia de educción se lleven a cabo. En el caso América Latina, se puede mencionar casos como el Espacio de América Latina y del Caribe-Unión Europea (ALCUE), el Espacio Iberoamericano del Conocimiento, y la Conferencia Regional de Educación Superior (CRES). Autores como Gacel-Ávila (2011), sostienen que para que el Proceso de Bolonia se extienda a la región y se pueda construir un espacio común de educación superior, se requiere de una mayor investigación acerca de la tipología institucional y la diversidad de los sistemas de educación superior, además de promover una reforma académica basada en estructuras académicas con un alto grado de innovación y modernización educativa, orientada a superar las debilidades existentes, como por ejemplo el desarrollo de competencias genéricas, las dificultades para el reconocimiento de grados, a fin impulsar un mayor nivel de movilidad de estudiantes, entre otras.

Ante este panorama, las instituciones de educación superior vienen desarrollando diferentes estrategias que les permitan afrontar los desafíos crecientes de un mundo cada vez más globalizado, y a falta de integración académica regional consolidada, han impulsado procesos como la movilidad estudiantil, vista como una estrategia de aprendizaje y cooperación educativa. Para autores como García (2013), la movilidad estudiantil internacional constituye "una expresión de la internacionalización de la educación superior, de la cooperación académica y la integración regional, además de ser un elemento clave para la mejora de la formación profesional, la movilización de saberes y la estructuración de redes de intercambio de conocimientos que permitan dar solución a los problemas de los países participantes" (p. 59). 
En esa misma línea, Theiler (2009) sostiene que la movilidad estudiantil ha favorecido los procesos de internacionalización de la educación superior, propiciando la generación de sistemas de cooperación interuniversitaria, así como, de integración regional.

La movilidad estudiantil estimula el crecimiento de los paises receptores, de acuerdo con Beine et al. (2014). Los estudiantes internacionales constituyen mano de obra altamente calificada para los países receptores, como consecuencia de ello algunos países han implementado políticas que favorecen la movilidad estudiantil; y la integración de estudiantes extranjeros en su mercado laboral. Entre las políticas de atracción de estudiantes se destacan: la flexibilización en la emisión de visas de estudiante en comparación a otros tipos de visados, subsidios para reducir el costo de las viviendas, transferencias de crédito, entre otros.

\section{Determinantes de la movilidad estudiantil}

Identificar los determinantes de la movilidad de estudiantes es fundamental para el diseño de políticas de internacionalización. De acuerdo con el Instituto Internacional de la UNESCO para la Educación Superior en América Latina y el Caribe - IESALC (2019), los principales determinantes de la movilidad internacional de los estudiantes son:

i) Factores educativos, estos se fundamentan en la diferencia de la calidad en la provisión de servicios educativos entre el país de origen y el país de destino, lo cual termina favoreciendo a la movilidad de estudiantes.

ii) Factores económicos, entre los que inciden más en la decisión de los estudiantes se encuentran: el nivel de desarrollo económico del país de destino, los costos asociados a la estadía y de estudios en la universidad de destino, y la tasa de retorno.

iii) Factor político- cultural, relacionado al perfil político y cultural del país de destino, en donde se puede destacar las características comunes como el tipo de religión; la estabilidad del país y el entorno.

iv) Factor Idioma, el idioma en el que se desarrollan los cursos y los aprendizajes se ha convertido en un factor esencial a la hora de determinar el lugar de destino.

En esa misma línea, Weisser (2016), sostiene que los principales factores que influyen en la movilidad estudiantil son la calidad institucional, la distancia y las condiciones económicas. Los estudiantes tienden a optar por los países con sistemas educativos de calidad; así como, por países que geográficamente sean más cercanos a sus países de origen y con características similares en lo cultural y en el idioma. Además, existe una fuerte correlación entre la movilidad de estudiantes y la perspectiva económica del país de destino.

Esta visión también es compartida por Rodríguez et al. (2010) quien, a partir de los hallazgos de estudios empíricos, sostiene que tanto el costo de vida y la distancia son factores relevantes al momento de explicar los flujos de movilidad estudiantil. Caruso y De Wit (2015), a través de estimaciones econométricas, determinaron que uno de los principales factores que influyen en la movilidad estudiantil en Europa, es la condición económica del país de destino o anfitrión, concluyendo por un lado que los países más ricos son más atractivos para los estudiantes, y que ello radica en el acceso a mayores oportunidades de empleo durante y después de la estadía. Por otro lado, su estudio resalta que cuanto más abierto es el país de destino, mayor es el número de entradas de estudiantes internacionales. Por su parte, Wei (2013), sostiene que el volumen del comercio de mercancías entre países facilita la movilidad internacional de estudiantes a través de las fronteras; y en el caso de los estudiantes internacionales de países en desarrollo, los factores educativos, así como económicos, tienen el mismo peso a la hora de determinar el país de destino. 
Alan Fairlie Reinoso

Algunos determinantes de la movilidad estudiantil universitaria de los países andinos

Autores como Abbott y Silles (2015), señalaron que la movilidad estudiantil ha sido impulsada, por un lado, por el crecimiento de la educación superior a nivel global, especialmente entre las economías de altos ingresos, y, por otro, por el valor percibido de matricularse en instituciones prestigiosas. En esa misma línea, el IESALC (2020), sostiene que el establecimiento de rankings, en especial nacionales, constituye una herramienta para garantizar la calidad de la educación superior y la autorregulación, al aumentar la competencia entre instituciones y programas.

De este modo, la importancia de la posición de las instituciones de educación superior dentro de los rankings nacionales e internacionales ha venido impactado significativamente en su imagen pública, aspecto que incide en la toma de decisiones de los estudiantes al momento de determinar el lugar de destino de sus intercambios. Por su parte, Gacel-Ávila (2017) sostiene que existe evidencia empírica que los Rankings en los estudiantes, especialmente en los estudiantes de posgrado, es un factor al momento de determinar la universidad o institución en donde realizarán sus estudios. Asimismo, señala que la presencia en los rankings globales tiende a incrementar el prestigio y la visibilidad de las instituciones de educación superior, por lo cual constituye una medida de reputación y posicionamiento.

\section{La movilidad estudiantil en América Latina}

La internacionalización de la educación superior en los países de América Latina, así como en la región Andina, presenta una tendencia creciente. Se ve a esta como una herramienta para mejorar la calidad académica de los programas educativos; además, de incrementar el prestigio y el perfil internacional de la institución.

De acuerdo, con los datos del IESALC, la movilidad estudiantil ha presentado un incremento constante tanto a nivel global, como dentro de la región. Es así como para el periodo comprendido entre el 2012-2017, el número de estudiantes latinoamericanos de educación superior fuera de su país pasó de 258 mil a 312 mil estudiantes. Pero, solo el 1,14\% del total de estudiantes en educación superior, realizaron estudios en el extranjero. En consecuencia, América Latina es la segunda región a nivel global donde la tasa de crecimiento de la movilidad ha sido la más baja (IESALC, 2019).

De igual forma, del total de estudiantes que realizaron estudios en el extranjero, solo el 38\% lo hizo en otros países de la región, donde Argentina es el país que acogió a más estudiantes. El 54\% restante optaron por países de Norteamérica y Europa Occidental. Sin embargo, existe un saldo negativo en lo que respecta a movilidad estudiantil en la región, es decir que son más los estudiantes que se trasladan al extranjero, que estudiantes extranjeros que ingresan a la región.

Es así como la apertura de movilidad internacional es una de las que más prevalecen en los países de la región. Según el British Council (2016), "los intercambios académicos, los programas de doble grado y la internacionalización de la acreditación, están siendo cada vez más usados por las universidades latinoamericanas para generar y mostrar mejoras en la calidad de sus programas y como parte de su proceso de internacionalización” (p.13).

Asimismo, Gacel-Ávila y Rodríguez-Rodríguez (2018b) destacan que en la región "las principales actividades de internacionalización son la movilidad de estudiantes” (p.65), la misma que se da con el afán de acceder a sistemas educativos de mayor calidad. Los hallazgos presentados en dicho estudio sostienen que del total de instituciones de educación superior que formaron parte del estudio, la gran mayoría consideró a la movilidad de estudiantes como la principal actividad de internacionalización, 
seguido de la movilidad de académicos y la participación en proyectos internacionales de cooperación al desarrollo. Además, resaltan que la movilidad internacional de estudiantes hacia el exterior es una de las más bajas en el mundo. En ese sentido, ambos autores identifican como principales obstáculos: la falta de dominio de idiomas por parte de los estudiantes, así como las dificultades administrativas o burocráticas.

Por otro lado, si bien existen iniciativas tanto al interior de las instituciones de educación superior, como iniciativas nacionales, bilaterales e incluso multilaterales a favor de la movilidad regional, estas no han tenido el efecto esperado. IESALC (2019), sostiene que "para promover la movilidad regional las iniciativas existentes no parecen tener la consistencia suficiente como para constituirse en un motor para la movilidad intrarregional"(p.37); puesto que, a pesar de tener elementos a favor como la proximidad, el tipo de cultura y mismo idioma, en la región no se ha trabajado para solucionar otros problemas estructurales como la calidad del sistema de educación del país o los costos asociados a estos, que son factores determinantes a la hora de escoger el país de destino.

Otro factor importante vinculado al flujo de estudiantes que realizan estudios en el extranjero es el reconocimiento de grados y títulos, ya que a nivel de la región no se cuenta con un acuerdo regional en dicha materia. Si bien los países andinos son miembros del Convenio Andrés Bello (CAB), y este en su Tratado Constitutivo señala en su artículo $5^{\circ}$ que los países que lo conforman reconocerán los grados y títulos que acrediten estudios académicos y profesionales expedidos por instituciones de educación superior de cada uno de ellos, no se han logrado consolidar avances en este ámbito.

Es así que dicho organismo en los últimos años ha desarrollado acciones orientadas a la construcción de espacios de trabajo e investigación dirigidos a conocer la estructura de los sistemas de educación superior en los países miembros, y los procesos de acreditación y evaluación de la calidad. Se busca identificar la contribución que desde la CAB se podría realizar en favor de la construcción de un Acuerdo Regional para el Reconocimiento de Títulos en Educación Superior; siendo una recomendación la mayor articulación e intercambio de experiencias con aquellas subregiones o acuerdos regionales que han avanzado más en el reconocimiento de títulos, como es el caso de Mercosur y Centroamérica a través del Consejo Superior Universitario Centroamericano-CSUCA- en Centroamérica (CAB, 2015). Pese a ello, a la fecha no se cuenta con avances concretos por parte de dicho organismo.

Situación similar se observó en cuanto a la aplicación del Convenio Regional de Convalidación de Estudios, Títulos y Diplomas de Educación Superior en América Latina y el Caribe del año 1974, el que a pesar de que fuera considerado como un hito "al asumir la equivalencia académica no como igualdad de contenidos sino como reconocimiento de un valor formativo similar" (IESALC, 2019. p 34) y ser firmado por 11 países latinoamericanos, este instrumento no ha estado operativo.

De acuerdo con Quinteros (2019), entre las principales limitantes que tenía el Convenio de Reconocimiento del 1974 que llevó a que no se aplique, se encuentran la falta de definición de los procesos de forma clara y precisa, ya que los conceptos de reconocimientos varían en cada uno de los países. Además, algunos países argumentaban la existencia de dificultades para la constatación de la legitimidad de las calificaciones académicas, y fiabilidad en torno a la calidad de los diplomas y títulos emitidos. Adicionalmente, no se habían especificado los requisitos para el reconocimiento y convalidación de los grados y títulos, a lo que se suma la falta de normas operativas que acompañen a las directrices del Convenio y que permitan la aplicación directa de este. Otro de los factores, es que el Convenio no discriminaba entre el reconocimiento con fines académicos, y el licenciamiento para el ejercicio profesional. 
Alan Fairlie Reinoso

Algunos determinantes de la movilidad estudiantil universitaria de los países andinos

En 2015, en el marco de la Reunión de Alto Nivel de Ministros de Educación en Brasilia, los Estados Miembros de la región decidieron elaborar un nuevo Convenio Regional, reconociendo la necesidad de establecer mecanismos para homologar los grados y títulos a nivel de la región, además de promover una mayor reciprocidad, en donde los acuerdos bilaterales y multilaterales tienen un rol fundamental. Ello llevó a que, el año 2019 en Buenos Aires, 23 países de la región (entre ellos los andinos) aprobaran y firmaran el nuevo Convenio Regional de Reconocimiento de Estudios, Títulos y Diplomas de Educación Superior en América Latina y el Caribe.

Este nuevo convenio favorecerá dinámicas de uniformidad, orden y seguridad para brindar una respuesta regional más sólida en cuanto al reconocimiento de estudios, títulos y diplomas en la región. Asimismo, constituye una herramienta que propiciará la movilidad académica dentro de la región, generando impactos positivos en materia de cooperación intrarregional, así como en la integración regional. También, promoverá la uniformización de los procesos de acreditación y con ello el atractivo conjunto de la región como destino receptor de movilidad (Quinteros, 2019).

Los Estados Parte signatarios de este convenio deberán adoptar las medidas necesarias para reconocer los estudios, títulos y diplomas de los países de la América Latina y el Caribe según los términos recogidos en este convenio y en concordancia con la normativa que rija en cada uno de sus países. Sin embargo, pese a que este convenio ha sido firmado por gran parte de los países de la región, se requiere que sea ratificado por lo menos 4 Estados miembros para que entre en vigor.

Es así como, los avances que se han dado en cuanto a la homologación y reconocimiento de grados y títulos han sido mayoritariamente través de los acuerdos bilaterales, bajo el principio de cooperación y reciprocidad. En el marco de los acuerdos binacionales se ha expresado la necesidad de establecer acuerdos mutuos de homologación y reconocimiento de títulos entre Perú, Bolivia, Ecuador y Chile.

Tabla 1: Reconocimiento de grados y títulos en los Gabinetes Binacionales

\begin{tabular}{|c|c|}
\hline Gabinetes Binacionales & Considerando \\
\hline $\begin{array}{l}\text { V Gabinete Binacional Perú- Bolivia } \\
\text { Declaración Presidencial de Ilo - } 2019\end{array}$ & $\begin{array}{l}\text { "32. Destacaron la pronta ratificación y entrada en } \\
\text { vigor del Convenio de Reconocimiento de Títulos } \\
\text { Profesionales y Grados Académicos de Educación } \\
\text { Superior Universitaria, que permitirá dar validez a los } \\
\text { certificados de estudios, títulos y grados académicos } \\
\text { de educación superior, reconocidos oficialmente por } \\
\text { los sistemas educativos de cada país, por medio de } \\
\text { sus respectivos organismos oficiales". }\end{array}$ \\
\hline $\begin{array}{l}\text { XIII Gabinete Binacional Perú - Ecuador } \\
\text { Declaración Presidencial de Tumbes -2019 }\end{array}$ & $\begin{array}{l}\text { "34. Señalaron la necesidad de continuar con la } \\
\text { definición de criterios comunes de homologación de } \\
\text { requisitos académicos, con miras a la suscripción del } \\
\text { convenio de reconocimiento de grados y títulos, lo } \\
\text { que beneficiará directamente a sus poblaciones". }\end{array}$ \\
\hline $\begin{array}{l}\text { III Gabinete Binacional Perú - Chile } \\
\text { Declaración Presidencial de Paracas -2019 }\end{array}$ & $\begin{array}{l}\text { "25. Acordaron, en materia de Educación, realizar } \\
\text { acciones que promuevan el intercambio de } \\
\text { conocimientos y material para el desarrollo de } \\
\text { programas de educación intercultural bilingüe e } \\
\text { implementación de acciones de capacitación. } \\
\text { Igualmente, se comprometieron a conformar una } \\
\text { Comisión Bilateral Técnica para abordar la } \\
\text { implementación del Acuerdo de Reconocimiento de } \\
\text { Títulos y Grados universitarios entre ambos países" }\end{array}$ \\
\hline
\end{tabular}

Fuente: Declaración Presidencial de Tumbes (2019), Declaración Presidencial de Ilo (2019), Declaración Presidencial de Paracas (2019). 
Teniendo en cuenta lo descrito, una de las prioridades de los países andinos debería ser garantizar la ratificación del Nuevo Convenio Regional para el Reconocimiento de Estudios, Títulos y Diplomas de Educación Superior en América Latina y el Caribe, auspiciado por la UNESCO, pues tal como lo señala el IESALC (2019), la movilidad estudiantil debe ser vista como un factor estratégico para dinamizar la conformación de nuevos espacios académicos regionales, y desarrollar un espacio latinoamericano del conocimiento; además de, promover un mayor acceso a la educación superior, así como el logro de los Objetivos de desarrollo sostenible (particularmente el ODS 4, educación de calidad).

\section{Restricciones al comercio de servicios educativos}

De acuerdo con la clasificación de la OMC, los servicios educativos se clasifican en: i) servicios de educación primaria; ii) servicios de educación secundaria; iii) servicios de educación superior; iv) servicios de educación de adultos; y v) otros. Existen varias maneras en que los servicios de educación mencionados pasan las fronteras a través de los proveedores de servicios de educación. Asimismo, la OMC estableció una tipología de las características de la internacionalización de los servicios en cuatro grandes áreas, que aplicadas a la educación se refieren a: la movilidad docente y estudiantil, la movilidad de instituciones, y la educación transfronteriza. En la actualidad, la investigación también es internacional.

En ese sentido, los servicios educativos se dan principalmente por la movilidad de estudiantes a través de las fronteras (Modo 2: consumo en el extranjero), seguido del movimiento de personas físicas (modo 4). Sin embargo, las nuevas tecnologías de la información están cambiando el panorama del comercio mundial de la educación. Estas, hacen posible la entrega de contenidos en formatos de audiovisuales a bajo costo, que ha llevado a un aumento del comercio de servicios educativos en el modo 1.

En cada caso se pueden identificar diferentes restricciones:

\section{Tabla 2: Lista de obstáculos al comercio de servicios de educación superior}

\begin{tabular}{|l|l|}
\hline $\begin{array}{l}\text { Modo 1: Suministro } \\
\text { transfronterizo }\end{array}$ & $\begin{array}{l}\text { - Restricción en la importación de material educativo producido electrónicamente } \\
\text { - Restricción en la transmisión electrónica del material del curso } \\
\text { - No reconocimiento de grados obtenidos a través del modo de distancia }\end{array}$ \\
\hline $\begin{array}{l}\text { Modo 2: Consumo } \\
\text { en el extranjero }\end{array}$ & $\begin{array}{l}\text { - Restricción en viajes al extranjero basados en disciplina o área de estudio } \\
\text { - Control de divisas (limitaciones) }\end{array}$ \\
\hline $\begin{array}{l}\text { Modo 3: Presencia } \\
\text { comercial }\end{array}$ & $\begin{array}{l}\text { - Insistencia en un socio local } \\
\text { - Insistencia en que el proveedor esté acreditado en el país de origen } \\
\text { - Insistencia en que el socio / colaborador sea de la corriente académica formal } \\
\text { - Insistencia en la igualdad de participación académica de los socios extranjeros y locales } \\
\text { - Desaprobación de operaciones de franquicia } \\
\text { - Restricciones en ciertas disciplinas / áreas / programas que se consideren estén en contra } \\
\text { de los intereses nacionales } \\
\text { - Limitaciones de la inversión extranjera directa por parte de los proveedores de educación } \\
\text { - Dificultades en la aprobación de negocios conjuntos }\end{array}$ \\
\hline $\begin{array}{l}\text { Modo 4: Presencia } \\
\text { de personas físicas }\end{array}$ & $\begin{array}{l}\text { - Restricciones de visado y entrada } \\
\text { - Requisitos de nacionalidad o residencia, idioma } \\
\text { - Restricción en la repatriación de ganancias }\end{array}$ \\
\hline
\end{tabular}

Fuente: Raychaudhuri (2007) 
Alan Fairlie Reinoso

Algunos determinantes de la movilidad estudiantil universitaria de los países andinos

De otra parte, las intervenciones del gobierno pueden limitar el comercio y la competencia en una o más de las siguientes maneras:

- mediante la restricción de entrada a un mercado,

- mediante la restricción de las operaciones y la flexibilidad de las empresas e instituciones,

- mediante la discriminación entre los proveedores nacionales y extranjeros, y / o entre la actividad interna y la exportación / importación operando de una manera no transparente. La aplicación de los reglamentos y las normas no pueden ser visibles, lo que aumenta los costos de cumplimiento.

Para Nyahoho (2011), el contenido actual del Acuerdo General sobre el Comercio de Servicios (AGCS) no aborda los obstáculos reales al comercio de servicios de educación: el aseguramiento de la calidad, la acreditación, el reconocimiento de diplomas y las visas de los visitantes, entre otras. En segundo lugar, a la luz de diversos factores de competitividad, las elecciones hechas por los países en el ámbito de la educación y el desarrollo son determinantes de la movilidad internacional de los estudiantes.

Por otro lado, autores como Tay (2014), consideran que la educación ha aumentado su participación entre los sectores de servicios, y en el Producto Interno Bruto (PIB) y empleo para muchas economías. En ese sentido, identifica como restricciones de los procesos de movilidad estudiantil, barreras comerciales (distancia, restricciones comerciales) y factores de preferencia (religión común, idioma común, frontera terrestre, idioma inglés, etc.). En la siguiente sección, se adapta el modelo de Tay (2014), para estimar las variables que explican la movilidad de estudiantes andinos hacia países desarrollados.

\section{Evidencia empírica}

\section{Especificación del modelo}

Siguiendo a Tay (2014), utilizaremos el modelo de gravedad aumentado para explicar los determinantes del comercio de la educación terciaria desde los países andinos hacia los países desarrollados para el periodo 2007-20151. En este modelo de gravedad aumentado se considera como variable dependiente el número de estudiantes andinos del nivel terciario $i$ que han cruzado territorio nacional con el propósito de obtener educación en los países desarrollados $j$ en el periodo $t\left(y_{i j t}\right)$. Es decir, se está haciendo referencia al modo 2 del comercio de servicios (consumo en el extranjero). Por otro lado, este modelo tiene como variables explicativas: el PBI del país de destino y del país de origen en el periodo $t\left(P B I_{j t}\right.$ y $\left.P B I_{i t}\right)$, la población del país de destino y del país de origen en el periodo $t\left(P_{j t}\right.$ y $\left.P_{i t}\right)$, la distancia entre el país de origen y el país de destino $\left(D_{i j}\right)$, el lenguaje común entre el país de origen y el país de destino $\left(L_{i j}\right)$, la religión común entre el país de origen y el país de destino $\left(R_{i j}\right)$, el índice de libertad económica del país desarrollado $j$ en el periodo $t\left(L_{j t}\right)$, y el índice de libertad económica del país andino $i$ en el periodo $t\left(L_{i t}\right)$. Cabe resaltar que estas dos últimas variables representan las barreras regulatorias de los países de destino y los países de origen ${ }^{2}$.

\footnotetext{
${ }^{1}$ En este trabajo, con excepción de Japón y Alemania (debido a que no se encuentra información para estos países), se consideran los mismos países exportadores que en el estudio de Tay (2014). Sin embargo, ellos consideran como países importadores a países desarrollados y en desarrollo.

${ }^{2}$ Cabe resaltar que estas barreras no son estrictamente barreras a la exportación o importación de servicios en educación terciaria.
} 


$$
\begin{gathered}
y_{i j t}=\beta_{1}+\beta_{2} P B I_{j t}+\beta_{3} P B I_{i t}+\beta_{4} P_{i j}+\beta_{5} P_{i t}+\beta_{6} D_{i j}+\beta_{7} L_{i j}+\beta_{8} R_{i j}+\beta_{9} L_{j t}+\beta_{10} L_{i t} \\
+\varepsilon_{i j t}
\end{gathered}
$$

Donde $\varepsilon_{i j t}$ es un proceso ruido blanco.

\section{Los datos y las variables}

En el modelo, los países de origen son Bolivia, Colombia, Ecuador y Perú ${ }^{3}(i=4)$, y los países de destino son Australia, Austria, Bélgica, Canadá, Dinamarca, España, Estados Unidos, Finlandia, Francia, Irlanda, Italia, Corea del Sur, Holanda, Noruega, Nueva Zelanda, Reino Unido, Suecia, Suiza y Turquía $(j=19)$. Y, el periodo de estudio es 2007-2015 $(t=9)$.

La variable endógena de 'número de estudiantes que han cruzado su territorio nacional con el propósito de obtener educación terciaria en un país desarrollado', se ha obtenido de las estadísticas mundiales de educación de la UNESCO ${ }^{4}$. Para las variables PBI 'por país de destino' y PBI 'por país de origen', se utilizó los datos de las cuentas nacionales del Banco Mundial y archivos sobre cuentas nacionales de la OCDE. Esta variable muestra el nivel de ingreso de los países en millones de dólares ${ }^{5}$ y servirá como proxy del tamaño de la economía de los países desarrollados y andinos. Para las variables 'población por país de destino' y 'población por país de origen', se utilizó la base de datos de Indicadores de Desarrollo del Banco Mundial'. Las variables 'distancia, lenguaje común y religión entre el país de origen y el país de destino' han sido obtenidas de la base proporcionada por Centre $d^{\prime} E$ tudes Prospectives et d'Informations Internationales (CEPII)'. La variable 'distancia' está medida como la distancia entre las capitales de los países en kilómetros ${ }^{8}$. La variable 'religión común' es un índice de escala 0-1 y ha sido construida a partir de la participación de cada una de las religiones en los países de origen y de destino. Esta variable será más cercana a uno mientras mayor sea la población de los países comparados que comparten las mismas religiones. Por otro lado, la variable 'lenguaje común' es medida a través de una variable dummy que toma el valor de 1 si el país andino y el país desarrollado tienen el mismo idioma oficial o el lenguaje primario, y 0 en cualquier otro caso. Finalmente, la variable 'índice de libertad económica para los países andinos y países desarrollados' ha sido obtenida a partir de la base de datos del Instituto Fraser'. Este índice tiene una escala de 0 a 10 y ha sido construido a partir de cinco indicadores: tamaño de gobierno ${ }^{10}$; estructura legal y seguridad de los

\footnotetext{
${ }^{3}$ Cabe precisar que para esta investigación se ha tomado como referencia los cuatro Países Miembros de la CAN: Bolivia, Colombia, Ecuador y Perú. Los cuales son estados miembros de este bloque de integración desde 1969 tras la suscrición del Acuerdo de Cartagena, no se toma en cuenta a Venezuela pues este país se retira la CAN en el 2011, fecha anterior al periodo de estudio de esta investigación. Asimismo, no se consideran a los cinco países asociados: Argentina, Brasil, Chile, Paraguay y Uruguay, pues estos no son miembros plenos de la CAN, aunque Chile si forma parte del Parlamento Andino. Véase: http://www.comunidadandina.org/Seccion.aspx?id=189\&tipo=QU\&title=somos-comunidad-andina

${ }^{4}$ Para más detalles, ver: http://data.uis.unesco.org

${ }^{5}$ Según Shepherd (2012) es mejor utilizar el PBI nominal ya que la delectación del PBI real estaría en términos de las resistencias multilaterales que representan índices de los precios no observables, generando resultados sesgados. Ver: Shepherd, Ben. (2012). The Gravity Model of International Trade: A User Guide. United Nations Economic and Social Commission for Asia and the Pacific, Bangkok.

${ }^{6}$ Véase: https://datos.bancomundial.org/indicador/sP.POP.TOTl

7 Véase: http://www.cepii.fr/CEPII/en/bdd_modele/bdd_modele.asp

${ }^{8}$ Esta variable ha sido construida a partir de la fórmula de los grandes círculos donde se utilizan las longitudes y latitudes entre las capitales de los países de origen y los países de destino.

${ }^{9}$ Véase: https://www.fraserinstitute.org/economicfreedom/dataset?geozone=world\&page $=$ dataset\&minyear $=2 \&$ max-year $=0 \&$ filter $=0$

${ }^{10}$ Este indicador toma en cuenta cuatro componentes: consumo del gobierno; transferencias y subsidios; empresas gubernamentales; e inversiones y tasa de impuesto marginal superior.
} 
Alan Fairlie Reinoso

Algunos determinantes de la movilidad estudiantil universitaria de los países andinos

derechos propiedad ${ }^{11}$; acceso a dinero ${ }^{12}$; liberalización del comercio internacional13; y regulación del crédito, trabajo y negocios ${ }^{14}$.

En la tabla $\mathrm{N}^{\circ} 3$ se presenta las estadísticas descriptivas de las variables utilizadas en la estimación. Como podemos observar en la tabla, el número promedio para la educación terciaria de estudiantes andinos hacia los países desarrollados para este periodo de estudio es, en promedio, de alrededor 514 estudiantes por año. Respecto a las variables explicativas, se destaca que el promedio del PBI de los países desarrollados es más de 10 veces el promedio del PBI de los países andinos. Asimismo, el promedio de la población de los países desarrollados es aproximadamente el doble del promedio de la población de los países andinos. Se resalta también que el índice de libertad económica es mayor para los países desarrollados que para los países andinos, aunque esta diferencia en el índice es de solamente un punto. Además, solo el 5\% de los países andinos comparte el mismo lenguaje que los países desarrollados. Cabe resaltar, que la variable endógena y la mayoría de las variables explicativas cambian en el tiempo, lo que ofrece mayor eficiencia en las estimaciones.

Tabla 3: Estadísticas descriptivas de las variables utilizadas en la estimación (promedio de los años 20072015)

\begin{tabular}{|c|c|c|c|c|c|c|}
\hline Variable & & Media & Std. Dev. & Mín. & Máx. & Observaciones \\
\hline \multirow{3}{*}{$\begin{array}{ll}\text { Movilidad } & \text { de } \\
\text { estudiantes } & \text { en } \\
\text { Educ. Superior } & \end{array}$} & overall & 514.356 & 1183.527 & 0 & 6899 & $\mathrm{~N}=684$ \\
\hline & between & & 1157.382 & 0.444 & 6620.127 & $\mathrm{n}=76$ \\
\hline & within & & 277.296 & -3185.200 & 2107.467 & $\mathrm{~T}=9$ \\
\hline \multirow{3}{*}{$\begin{array}{l}\text { PBI del país de } \\
\text { destino (millones) }\end{array}$} & overall & 1750000 & 3320000 & 142000 & 16700000 & $\mathrm{~N}=684$ \\
\hline & between & & 3340000 & 153000 & 15500000 & $\mathrm{n}=76$ \\
\hline & within & & 155000 & 897000 & 2970000 & $\mathrm{~T}=9$ \\
\hline \multirow{3}{*}{$\begin{array}{l}\text { PBI del país de } \\
\text { origen (millones) }\end{array}$} & overall & 140000 & 110000 & 17200 & 360000 & $\mathrm{~N}=684$ \\
\hline & between & & 109000 & 21100 & 307000 & $\mathrm{n}=76$ \\
\hline & within & & 20400 & 95300 & 193000 & $\mathrm{~T}=9$ \\
\hline \multirow{3}{*}{$\begin{array}{l}\text { Población del país } \\
\text { de destino (miles) }\end{array}$} & overall & 42300 & 67800 & 4224 & 321000 & $\mathrm{~N}=684$ \\
\hline & between & & 68200 & 4386 & 311000 & $\mathrm{n}=76$ \\
\hline & within & & 1658 & 32200 & 51700 & $\mathrm{~T}=9$ \\
\hline \multirow{3}{*}{$\begin{array}{l}\text { Población del país } \\
\text { de origen (miles) }\end{array}$} & overall & 25400 & 14200 & 9441.444 & 48200 & $\mathrm{~N}=684$ \\
\hline & between & & 14200 & 10100 & 46400 & $\mathrm{n}=76$ \\
\hline & within & & 882.879 & 23400 & 27200 & $\mathrm{~T}=9$ \\
\hline Distancia & overall & 10197.940 & 2391.492 & 3815.184 & 17663.5 & $\mathrm{~N}=684$ \\
\hline
\end{tabular}

11 Este indicador tiene nueve componentes: independencia judicial, cortes imparciales, protección a los derechos de propiedad, interferencia militar en el Estado, integridad del sistema legal, cumplimiento de los contratos, costos regulatorios de la venta de bienes inmuebles, apoyo en la policía, y costos comerciales del crimen.

12 Este indicador tiene cuatro componentes: crecimiento del dinero, desviación estándar de la inflación, inflación (en los años más recientes), y libertad para poseer cuentas bancarias en moneda extranjera.

${ }^{13}$ Este indicador posee cuatro componentes: aranceles, barreras al comercio regulatorias, tasas de intercambio en el mercado negro, y control del movimiento de capital y mano de obra.

${ }^{14}$ Este indicador tiene tres componentes: regulación de mercado de crédito, regulación del mercado de trabajo, $y$, regulaciones comerciales. 


\begin{tabular}{|c|c|c|c|c|c|c|}
\hline & between & & 2405.622 & 3815.184 & 17663.5 & $\mathrm{n}=76$ \\
\hline & within & & 0 & 10197.940 & 10197.94 & $\mathrm{~T}=9$ \\
\hline \multirow{3}{*}{ Lenguaje común } & overall & 0.053 & 0.223 & 0 & 1 & $N=684$ \\
\hline & between & & 0.225 & 0 & 1 & $\mathrm{n}=76$ \\
\hline & within & & 0 & 0.053 & 0.053 & $\mathrm{~T}=9$ \\
\hline \multirow{3}{*}{ Religión común } & overall & 0.392 & 0.342 & 0.001 & 0.936 & $N=684$ \\
\hline & between & & 0.344 & 0.001 & 0.936 & $\mathrm{n}=76$ \\
\hline & within & & 0 & 0.392 & 0.392 & $\mathrm{~T}=9$ \\
\hline \multirow{3}{*}{$\begin{array}{l}\text { Libertad económica } \\
\text { del país de destino }\end{array}$} & overall & 7.693 & 0.388 & 6.540 & 8.480 & $\mathrm{~N}=684$ \\
\hline & between & & 0.377 & 6.846 & 8.410 & $\mathrm{n}=76$ \\
\hline & within & & 0.100 & 7.387 & 8.056 & $\mathrm{~T}=9$ \\
\hline \multirow{3}{*}{$\begin{array}{l}\text { Libertad económica } \\
\text { del país de origen }\end{array}$} & overall & 6.589 & 0.614 & 5.660 & 7.640 & $\mathrm{~N}=684$ \\
\hline & between & & 0.610 & 5.909 & 7.528 & $\mathrm{n}=76$ \\
\hline & within & & 0.102 & 6.340 & 6.770 & $\mathrm{~T}=9$ \\
\hline
\end{tabular}

Fuente: Elaboración propia en base a UNESCO, Banco Mundial, CEPPI y Freaser Institute.

\section{Resultados econométricos}

En la Tabla 4 se presenta la estimación de tres modelos mediante la metodología MCO de datos de panel, que es conocido como el Modelo Pooled ${ }^{15}$. Siguiendo el modelo de Tay (2014), la variable endógena y todas las variables explicativas, con excepción de lenguaje y religión comunes, están en logaritmos ${ }^{16}$.

En la estimación del modelo (a), la variable más importante para explicar la movilidad estudiantil en educación terciaria desde países andinos hacia países desarrollados es el nivel de ingresos de los países de destino. Un aumento de 1\% en el PBI de los países de destino, aumenta, en promedio, 2,27\% la movilidad de estudiantes de educación terciaria desde países andinos hacia países desarrollados. Esto significa que los estudiantes de educación terciaria de países andinos prefieren a los países de destino que tienen mayores ingresos, probablemente esto se encuentre relacionado con las mayores oportunidades laborales durante y después del periodo de estudio. Por otro lado, el impacto del nivel de ingresos en los países andinos también aumenta la movilidad de estudiantes de educación terciaria, sin embargo, el impacto es aproximadamente cinco veces menor. Un aumento de $1 \%$ en el PBI de los países andinos, aumenta, en promedio, en solo 0,4\% la movilidad de estudiantes de educación terciaria. Cabe señalar que este impacto es significativo al 10\% de nivel de significancia. En este modelo también se muestra que la variable distancia es negativa y significativa al $10 \%$, lo que nos dice que mientras mayor sea la distancia entre la capital del país de origen y la capital del país de destino, menor será la movilidad de estudiantes andinos hacia países desarrollados. Asimismo, en este mismo modelo se observa también una relación negativa y altamente significativa entre la población de destino y la movilidad estudiantil. Esto significa que una mayor población de un país exportador

15 Cabe señalar que los resultados en los tres modelos son robustos a heteroscedasticidad y autocorrelación. Asimismo, mediante el test VIF, se descartó la presencia de multicolinealidad en el modelo.

${ }^{16}$ La estimación incluye 27 observaciones menos debido a la presencia de ceros en la variable endógena. 
Alan Fairlie Reinoso

Algunos determinantes de la movilidad estudiantil universitaria de los países andinos

respecto a otro país exportador posee una menor oferta de servicios de educación terciaria conllevando a una reducción de la movilidad estudiantil.

Si se incluyen las variables lenguaje y religión comunes, modelo (b), el nivel de ingresos de la economía de destino sigue siendo la variable más importante para explicar la movilidad de estudiantes en educación terciaria. Sin embargo, el lenguaje común tiene un impacto grande, significativo y muy parecido al impacto que tiene la variable PBI del país de destino sobre la movilidad en este modelo. Si un país andino y un país desarrollado comparten el mismo idioma, la movilidad estudiantil desde países andinos a países desarrollados aumenta en 2,9\%. Por otro lado, la variable religión común, tiene un impacto positivo y significativo, lo que significa que mientras mayor sea la población en el país de origen y país de destino que comparten la misma religión, mayor será movilidad estudiantil. Las demás variables explicativas del modelo (b), con excepción de distancia, tienen un comportamiento similar a la del modelo (a). Llama la atención que la variable distancia en el modelo (b) tenga un impacto positivo y significativo sobre la movilidad estudiantil, no obstante, según Dee (2008) es posible que el impacto de la distancia tenga un impacto indeterminado. Respecto a la bondad de ajuste del modelo, podemos observar que el modelo (b), presenta un ajuste superior en un $16 \%$ respecto al modelo (a).

Si se incluyen las barreras regulatorias de los países de origen y de destino, modelo (c), podemos observar que la variable más importante para explicar la movilidad estudiantil terciaria ya no es el ingreso de los países de destino, sino son las barreras regulatorias de los países exportadores. Un aumento de una unidad adicional en el índice de libertad económica del país de destino aumenta en 4,48\% la movilidad de estudiantes andinos hacia países desarrollados. Asimismo, la barrera regulatoria en el país de origen también explica la movilidad de estudiantes en educación terciaria, aunque su impacto es menor y solo es significativa en un 10\%. Un aumento de una unidad adicional en el índice de libertad económica reduce la movilidad estudiantil en 1,1\%. Esto es consistente con la teoría del efecto pulling-back que se señala en Tay (2014).

Una limitación del modelo es la posible endogeneidad de los regresores, conllevando a que los resultados sean sesgados e inconsistentes. Sin embargo, pensamos que esto no se debe a una doble causalidad, porque es poco probable que la movilidad de estudiantes andinos a países desarrollados influya sobre el PBI, distancia, lenguaje común, religión común o sobre las restricciones a las importaciones y exportaciones. Respecto a variables omitidas, se ha estimado el test de error de especificación de Ramsey mostrando evidencia de que el modelo está bien especificado. La gran cantidad de datos (657 observaciones) y la buena bondad de ajuste del modelo pooled (80\%) nos hace pensar que los resultados mediante el modelo Pooled son los correctos.

Tabla 4: Estimación del efecto de las barreras al comercio de la movilidad estudiantil de educación superior desde los países andinos hacia los países desarrollados (panel 2007-2015)

\begin{tabular}{llll}
\hline & (a) & (b) & (c) \\
$\ln ($ PBI origen) & $0.421^{*}$ & $0.501^{* * *}$ & $0.378^{* *}$ \\
& {$[0.228]$} & {$[0.171]$} & {$[0.190]$} \\
& & & \\
$\ln ($ PBI destino) & $2.274^{* * *}$ & $3.034^{* * *}$ & $2.944 * * *$ \\
& {$[0.256]$} & {$[0.182]$} & {$[0.161]$} \\
$\ln ($ distancia $)$ & $-0.409^{*}$ & $0.531^{* * *}$ & $0.849^{* * *}$
\end{tabular}




\begin{tabular}{|c|c|c|c|}
\hline & {$[0.215]$} & {$[0.175]$} & [0.179] \\
\hline $\begin{array}{l}\ln \text { (población de } \\
\text { origen) }\end{array}$ & $\begin{array}{c}0.428 \\
{[0.388]}\end{array}$ & $\begin{array}{l}0.367 \\
{[0.291]}\end{array}$ & $\begin{array}{l}0.693 * * \\
{[0.351]}\end{array}$ \\
\hline $\begin{array}{l}\ln \text { (población de } \\
\text { destino) }\end{array}$ & $\begin{array}{l}-0.822 * * * \\
{[0.232]}\end{array}$ & $\begin{array}{l}-1.533^{* * *} \\
{[0.154]}\end{array}$ & $\begin{array}{l}-1.382^{* * *} \\
{[0.135]}\end{array}$ \\
\hline Lenguaje Común & & $\begin{array}{l}2.978^{* * *} \\
{[0.144]}\end{array}$ & $\begin{array}{l}3.034^{* * *} \\
{[0.149]}\end{array}$ \\
\hline Religión común & & $\begin{array}{l}1.193 * * * \\
{[0.142]}\end{array}$ & $\begin{array}{l}1.334 * * * \\
{[0.151]}\end{array}$ \\
\hline $\begin{array}{l}\text { ln (liberación } \\
\text { económica país de } \\
\text { origen) }\end{array}$ & & & $\begin{array}{l}-1.075^{*} \\
{[0.597]}\end{array}$ \\
\hline $\begin{array}{l}\text { ln (liberación } \\
\text { económica país de } \\
\text { destino) }\end{array}$ & & & $\begin{array}{l}4.484^{* * *} \\
{[1.050]}\end{array}$ \\
\hline $\mathrm{N}$ & 657 & 657 & 657 \\
\hline R-cuadrado & 0.63 & 0.79 & 0.80 \\
\hline
\end{tabular}

\section{Conclusiones}

En este documento, se determinan los principales factores que influyen en los estudiantes de la región Andina para realizar estudios en el extranjero, y por ende en la selección del país de destino. Se identifican las principales modalidades empleadas dentro del proceso de internacionalización, resaltando que estas se sustentan en las asimetrías que definen la relación entre los países más y menos desarrollados, en un contexto de creciente demanda de formación de mayor calidad.

Por lo cual, de los diferentes modelos de internacionalización, se estudia el modo 2 del AGCS referido a la movilidad estudiantil. Teniendo en cuenta ello, se hace una revisión de la literatura respecto a la importancia de la movilidad estudiantil como un eje de los procesos de internacionalización, señalando que esta no solo permite proyectar a las universidades, sino que prestigia al país, y mejora su inserción internacional, por lo cual existen factores tanto internos (institucionales) como externos (entre países), que la facilitan.

La literatura identifica cuatro factores que son determinantes al momento en el que los estudiantes internacionales seleccionan sus países de destino, entre los que podemos destacar: factores educativos, económicos, político- cultural, y de idioma. Factores que también se encuentran en los países de América Latina, y han limitado el potencial que tiene la movilidad estudiantil como estrategia en los procesos de internacionalización de la educación superior. A esto se suma que hay 
Alan Fairlie Reinoso

Algunos determinantes de la movilidad estudiantil universitaria de los países andinos

disposiciones que restringen la competencia de instituciones extranjeras, así como el reconocimiento de títulos, que constituyen problemas estructurales en la región.

En ese sentido, y tomando como base el modelo de Tay (2014), se estimó un modelo de gravedad aumentado para explicar los determinantes del comercio de la educación terciaria desde los países andinos hacia los países desarrollados para el periodo 2007-2015, partiendo de tres estimaciones con variables diferentes. En el primer caso, la variable más importante para explicar la movilidad estudiantil es el ingreso del país receptor, encontrando que un aumento de 1\% en el PBI de los países de destino, aumenta en promedio 2,27\% la movilidad de estudiantes de educación terciaria desde países andinos hacia los países desarrollados, asociado muchas veces a mayores oportunidades laborales.

En la segunda estimación, se añaden las variables lenguaje y religión comunes. Si bien la movilidad estudiantil desde países andinos a países desarrollados aumenta en 2,9\% en caso ambos países compartan un mismo idioma, el ingreso en la economía del país de destino sigue siendo la más relevante, pero el lenguaje común adquiere un impacto grande, similar a la anterior. Estos resultados econométricos permiten corroborar y comprobar lo señalado por Weisser (2016), Caruso y De Wit (2015), Rodríguez et al. (2010), en sus respectivos estudios.

Finalmente, en la tercera estimación, se añade una variable que incorpora las barreras regulatorias de los países (índice de libertad económica), es así como aumento de una unidad adicional en el índice de libertad económica del país de destino, aumenta en 4,48\% la movilidad de estudiantes andinos hacia países desarrollados. En este caso, las barreras en los países de destino pasan a ser la variable más importante en la estimación. Esta variante en el modelo nos permite ratificar lo señalado por Caruso y De Wit (2015) Wei (2013), quienes sostienen que una mayor apertura, propicia un mayor flujo de estudiantes internacionales.

Así, independientemente de las restricciones internas (económicas, idioma), se identifican también restricciones externas, referidas a barreras regulatorias. Esto sugiere, que convenios con países donde hay más apertura y menores regulaciones, favorecen la movilidad. Más allá de variables económicas, la movilidad depende de factores de atracción como la calidad de las instituciones y obstáculos como el idioma y reconocimiento de títulos. Se requiere identificar además como influyen otras barreras comerciales, regulatorias e institucionales, lo que se debe hacer en próximos trabajos.

En el caso de los obstáculos externos, se puede señalar como uno de los principales factores la dificultad que tienen los estudiantes para el reconocimiento de títulos y cursos, que no solo dependen de las universidades, sino del marco legal vigente a nivel nacional y entre países, y si pertenecen a un acuerdo de integración regional.

Los hallazgos de este artículo identifican algunos determinantes de la movilidad estudiantil de nuestros países, a otros desarrollados. Aparte de buscar otras barreras existentes, para tratar de removerlas en convenios bilaterales, deberíamos profundizar relaciones de cooperación entre países andinos, y los acuerdos al interior de los procesos de integración de los que formamos parte. En ese sentido, se deben diseñar políticas domésticas y buscar en los acuerdos de integración regional una coordinación e integración regional de la educación superior, para lo cual se necesita consolidar los avances en Mercosur, Alianza del Pacífico, Comunidad Andina y otras regiones latinoamericanas, de forma tal que se defina una estrategia territorial integral que supere las limitaciones de los esfuerzos, que, hasta el momento, han sido principalmente al interior de cada bloque. 
Para las universidades andinas, deben plantearse alianzas estratégicas con instituciones de la CAN y la Alianza del Pacífico, fortaleciendo además los mecanismos de los acuerdos de integración regional para acreditación, reconocimiento de títulos, doble titulación, movilidad, etc. También es factible con otras universidades del Mercosur, en el ámbito sudamericano. Un segundo nivel de alianza es el espacio euro-latinoamericano. Una alianza con universidades europeas en áreas específicas puede ser mutuamente conveniente, ya que es una puerta de entrada para nosotros en Europa con la globalización de algunas áreas, pero también una opción interesante para ellas, donde la cooperación se puede profundizar en diferentes planos.

Teniendo en cuenta ello, resulta indispensable que los países de la región Andina ratifiquen el nuevo Convenio Regional de Reconocimiento de Estudios, Títulos y Diplomas de Educación Superior en América Latina y el Caribe, pues es una herramienta fundamental para la revitalización de la movilidad regional, en especial la intrarregional, así como para promover los procesos de internacionalización y mejorar de la calidad de las instituciones de educación superior y promover la integración regional. Asimismo, se debe propiciar el intercambio de experiencias sobre todo de aquellas regiones o bloques de integración que han avanzado en el establecimiento de mecanismos para facilitar y promover la movilidad estudiantil y la homologación de grados y títulos, como es el caso del Mercosur que cuenta con una agenda de trabajo para la integración educativa amplia que ha llevado al establecimiento del Acuerdo sobre Reconocimiento de Títulos de Grado de Educación Superior del MERCOSUR. Este Acuerdo permite validar los diplomas de los países del bloque, teniendo en cuenta que los títulos universitarios acreditados por ARCUSUR (el sistema regional de acreditación universitaria), y que se encuentra en proceso de ratificación para entrar en vigencia.

En esa misma línea, se debe continuar promoviendo cooperación interregional e intrarregional para facilitar la movilidad estudiantil, y poder afrontar los diferentes factores que limitan la movilidad estudiantil, siendo un mecanismo para ello los acuerdos establecidos en los Gabinetes Binacionales, en donde los países andinos, así como Chile han mostrado su voluntad para lograr avances concretos en la materia que deben de ser fortalecidos y encaminados.

Por otro lado, y teniendo en cuenta que la pandemia del COVID-19 ha impactado significativamente en el flujo de la movilidad de estudiantes y, tal como lo sostiene Marchesini (2020), las restricciones de movilidad y los efectos a causa de esta ha generado que las instituciones de educación superior cambien su enfoque de internacionalización y surjan nuevas opciones, como el impulso de la movilidad virtual, el co-teaching internacional, entre otros. Ello implica que el modo 1 del AGCS tenga un mayor protagonismo. Por lo tanto, la modalidad a distancia, virtual, podría ser una alternativa para hacer frente a los desafíos propios de la pandemia, además de constituir una forma de extensión y digitalización del modelo universitario, permitiendo potenciar su componente internacional, ya que proporciona acceso a programas internacionales sin los costos de movilidad física. Pero, esto supone desarrollar la calidad en los materiales y contenidos, competitivos internacionalmente y adopción y manejo de plataformas virtuales. Allí se encuentra el principal reto de los países de la región andina, por lo menos en el corto plazo. 
Alan Fairlie Reinoso

Algunos determinantes de la movilidad estudiantil universitaria de los países andinos

\section{Referencias}

Abbott, A., y Silles, M. (2016). Determinants of international student migration. The World Economy, 39(5), 621-635.

Beine, M., Noël, R., y Ragot, L. (2014). Determinants of the international mobility of students. Economics of Education Review, 41, 40-54.

British Council (2016), La reforma del sistema universitario peruano: internacionalización, avance, retos y oportunidades.

CAB (2015) Construcción de un Espacio Latinoamericano para la Educación Superior Un aporte a la discusión Regional

Caruso, R y De Wit, H (2015) Determinants of Mobility of Students in Europe: empirical evidence for the period 1998-2009, Revista de Estudios en Educación Internacional, 19 (3), 265-282.

Comisión Europea (23.03. 2021) Proceso de Bolonia y Espacio Europeo de Educación Superior. Comisión Europea.https://ec.europa.eu/education/policies/higher-education/bologna-process-andeuropean-higher-education-area_es

Dee, P. (2008). International Student Movements and the Effects of Barriers to Trade in Higher Education Services. Australian National University. February,2008.

Gacel-Ávila, J y Rodrigues-Rodrigues, S. (2018a). La internacionalización de la educación terciaria en América Latina y el Caribe: avances, tendencias y visión a futuro. Educación superior, internacionalización e integración en América Latina y el Caribe-balance regional y prospectiva. Caracas: UNESCO-IES ALC y Córdoba: Universidad Nacional de Córdoba.

Gacel-Ávila, Jocelyne, y Rodríguez-Rodríguez, Scilia. (2018b). Internacionalización de la educación superior en América Latina y el Caribe: un balance, México, UNESCO-IESALC

Gacel-Ávila, J. (2017). Estrategias de internacionalización de la educación superior: implementación,evaluación y rankings. Caracas: UNESCO-IESALC.

Gacel-Ávila, J (2011). Impacto del proceso de Bolonia en la educación superior de América Latina. En: Globalización e internacionalización de la educación superior]. Revista de Universidad y Sociedad del Conocimiento (RUSC). Vol. 8, n. ${ }^{\circ}$ 2, 123-134

García Palma, J. J. (2013). Movilidad estudiantil internacional y cooperación educativa en el nivel superior de educación. Revista Iberoamericana De Educación,61, 59-76. https://doi.org/10.35362/rie610600

IESALC (2020) La garantía de calidad y los criterios de acreditación en la educación superior: Perspectivas internacionales.

IESALC (2019) La Movilidad en la Educación Superior en América Latina y El Caribe: Retos y Oportunidades de un Convenio Renovado para el Reconocimiento de Estudios, Títulos y Diplomas. Caracas.

Knight, J. (2003). Updated definition of internationalization. International higher education

Marchesini, G (2020) COVID-19 and Internationalization in the MENA Region

Nyahoho, E. (2011). International Trade in Education Services: Its Scope and Challenges Associated with Liberalization. Journal of Economic and Social Policy, 14(1), 9.

Organización Mundial de Comercio (2015). Entender a la OMC. Ginebra

Quinteros (26 de noviembre 2019).El Nuevo Convenio de Reconocimiento de Estudios, Títulos y Diplomas de Educación Superior. Conferencia UNESCO-IESALC. Disponible en https://www.youtube.com/watch?v=jpQXpdtqaX0

Rama, C. (2014). Las Diversas Internacionalizaciones de la Educación Superior en América Latina.

Raychaudhuri, A., y De, P. (2007). Barriers to trade in higher education services: Empirical evidence from Asia-Pacific countries. Asia-Pacific Trade and Investment Review, 3(2), 67-88. 
Rodríguez González, C., Bustillo Mesanza, R., y Mariel, P. (2010). The Determinants of International Student Mobility Flows: An Empirical Study on the Erasmus Program. Higher Education, 62(4), 413-430.

Shepherd, Ben. (2012). The Gravity Model of International Trade: A User Guide. United Nations Economic and Social Commission for Asia and the Pacific, Bangkok

Tay, C. (2014). An econometric model on bilateral trade in education using an augmented gravity model. Journal of Industrial Engineering and Management (JIEM), 7(2), 401-412.

Theiler, J (Setiembre 2019), La UNL y su politica de movilidad estudiantil a lo largo de 20 años, En el $1^{\circ}$ Congreso de Internacionalización de la Educación Superior, Brasil.

Theiler, J (2009). Programas de Movilidad Internacional, su organización y las buenas prácticas para su gestión y administración. Universidad Nacional del Litoral. Argentina.

Wei, H. (2013). An empirical study on the determinants of international student mobility: A global perspective. Higher Education, 66, 105-122.

Weisser, R. (2016). Internationally mobile students and their post-graduation migratory behavior: An analysis of determinants of student mobility and retention rates in the EU. In OECD Social, Employment and Migration Working Papers (Vol. 186). Paris: OECD Publishing.

(c) (1) (9)

Open Access This article is licensed under a Creative Commons Attribution-Non Commercial 4.0 International License, which permits the use, adaption and sharing as long as you give appropriate credit to the original author(s) and the source. The images or other third party material in this article are included in the article's Creative Commons license, unless indicated otherwise in a credit line to the material. If materials are not included in the article's Creative Commons license and your intended use is not permitted by statutory regulation or exceeds the permitted use, you will need to obtain permission directly from the copyright holder.

To view a copy of this license, visit http://creativecommons.org/licenses/by-nc/4.0/.

(C) The Author(s) 2021. 